\title{
The Macau Family-in-Transition: The Perceived Impact of Casino Employment on Family Relationships Among Dealer Families
}

\author{
Simon T.M. Chan ${ }^{*}$, Clara L.Y. Kwok and Johnny Y.N. Siu \\ Department of Social Work, Hong Kong Baptist University, Hong Kong
}

\begin{abstract}
Although there is growing consideration of the negative impacts that are instigated by the booming gaming industry on the physical health and job satisfaction of casino employees in Macau, a critical research gap exists in the understanding of the perceived impacts of casino employment on the family life of the dealers. By drawing on evidence from a qualitative study carried out in Macau, the specifics of the changes in the family lives among dealer families and how family relationships have been affected by casino employment will be explored and analyzed in this paper. With a focus-group-interview design, 113 casino dealers have been interviewed in terms of the impacts of casino employment on their family relationships. The findings suggest that while there has been undeniably a positive change in terms of the financial situation of the family, this has neither improved family bonding, nor led to a large-scale transformation of family relations in the direction of greater harmony and functionality. Yet even though there are conflicting views on the perceived impacts of casino employment on their spousal, parental and overall family relationships, the study participants generally agree that the fatigue and incompatibility of schedules that are resultant of casino work impose significant challenges in their parental role. The paper concludes with recommendations to address the issues found in this study.
\end{abstract}

Keywords: Family, Macau, dealers, casino, family relationship.

\section{INTRODUCTION}

Gambling has recently become an area of considerable academic and general interest. This is resultant of the many opportunities for gambling activities on an international scale, such as horseracing, football betting and casino gaming. Gambling has become synonymous with cities such as Las Vegas and Macau, which feature numerous casinos. The residents of such cities have internalized the idea that the gaming industry promotes employment and tourism opportunities and alleviates poverty [1].

Consequently, the influence of casino work from the perspective of casino dealers in Macau will be examined in this paper. A theoretical framework which incorporates the work-family conflict model in Greenhaus and Beutell [2] will be applied. This new perspective may provide insights into the family problems associated with the gaming industry. The first objective of this paper is to explore how the work of these casino dealers affects their families, with particular focus on spousal and parent-child relationships, as well as relationships with other family members. The second objective, based on the findings of the first objective, is to provide possible proposals for casino enterprises, the Macau Special Administrative Region (MSAR) Government, general public and social service sector to address the needs of dealers and their family members.

*Address correspondence to this author at the Hong Kong Baptist University, Room AAB 1022, 10/F, Academic and Administrative Building, 15 Baptist University Road, Baptist University Road Campus, Kowloon Tong, KLN, Hong Kong; Tel: +852-3411 7142;

E-mail:simon@hkbu.edu.hk

\section{MONTE CARLO OF THE ORIENT}

Macau stretches from the southeast coast of China to the western bank of the Pearl River Delta. It was under the administration of the Portuguese from the 16th century until December 20, 1999, when it was handed back to the People's Republic of China. After the handover, Macau was established as a special administrative region (SAR) of China. According to the Macao Special Administrative Region Statistics and Census Service, the total population of Macau has increased from 448,495 in 2003 [3] to 607,500 in 2013 [4]. The majority of the citizens in Macau are Chinese (i.e., about $95 \%$ ) with only $3 \%$ Portuguese; therefore, a certain amount of assimilation between the Chinese and the Portuguese is expected. The distinct history of Macau has played an influential role in the formation of its unique culture, resultant of the fusion of the Portuguese and Chinese cultures.

\section{GAMING INDUSTRY IN MACAU}

In addition to the change in sovereignty, the policy of releasing gaming licenses in Macau has had a significant effect on its economy and society as a whole. The gaming industry had operated under a government monopoly from 1962, but in 2002, the MSAR government ended tradition and released gaming licenses to two private companies. Consequently, many casino operators who hailed from Las Vegas then attempted to enter the market. The number of casinos rose from 11 to 33 [5] in the wake of the decision by the government to grant gaming licenses to the private sector. The increasing number of casinos has allowed the gaming industry to flourish, thus making a significant contribution to the economy of Macau. The casinos have 
attracted more tourists worldwide, increased tax revenues, and turned the tourism and gaming industries into the main sources of revenue for Macau. In 2004, the revenue generated by the gaming industry was US\$5.49 billion, and has dramatically increased in the past few years, reaching more than US\$17.59 billion in 2013 [4].

The increasing number of casinos and the prominence of the gaming industry have attracted more people to participate in gambling, which has resulted in negative impacts. According to Macau police data, more than $31 \%$ of the reported crimes are directly related to gambling [6]. Increasingly more individuals have also been attracted to work in the gaming or related industries. According to data from the Statistics and Census Service of the MSAR Government [4], 83,300 individuals were employed in gaming industry-related positions in 2013. There has been a continuous growth in the number of such employment since the restriction on the gaming industry was relaxed in 2002 . Gaming employees constituted $23.07 \%$ of the total work population in 2013 [4].

\section{CASINO EMPLOYEES IN MACAU}

As indicated above, the tourism and gaming industries are the main sources of revenue for Macau. Casinos operate 24 hours a day, and to maintain round-the-clock operations, it is vital to have large numbers of employees in shift duties. Although several studies have targeted casino employees, their main focus has been on work-related issues, such as stress [7], job satisfaction [8], gambling behavior [9, 10] and the work environment [11]. Researchers at the Macau Polytechnic Institute interviewed 873 casino employees to investigate their sources of stress [7] and found that 619 participants $(70.9 \%)$ feel stressed by their work. The sources of stress were attributed to the management system (47.4\%), customers $(43 \%)$ and long work hours (36.9\%). Dangerfield [8] reported that casino employees are neither satisfied with their work nor their work environment. It could therefore be insinuated that high levels of stress and dissatisfaction at work may have an influence on the relationships of casino employees with their family members, although previous research has rarely taken this into consideration.

\section{JOB NATURE OF DEALERS AND IMPACTS ON FAMILY}

In this paper, the focus is on casino dealers as they are predominant among the casino employees and the nature of their job involves intensive frontline work. Dealers are responsible for explaining and enforcing the rules of the games, conducting the games and handling money transactions with customers. They are the most high-profile workers in casinos, and have direct interactions with customers. Workplace harassment, for instance, verbal or physical bullying from the players, has effects on the psychological and physical well-being of the dealers. As mentioned above, the sources of stress are not only due to the frontline nature of the work, but also the shift work.

An irregular work schedule reduces the time that dealers spend with their families, despite their personal desires and needs. It can be stressful to balance work commitments with family relationships, and this type of stress may also influence marital relationships. Our hypothesis that family relationships are affected by casino work accords with the model of work-family conflict proposed by Greenhaus and Beutell [2].

According to this model, work-family conflict exists when (1) time devoted to the requirements for one role makes it difficult to fulfill the requirements of another; (2) the strain from participation in one role causes difficulties in fulfilling the requirements of another; and (3) the specific behavior required by one role creates challenges in fulfilling the requirements of another role. Casino dealers may face these three types of conflicts between work and family.

Shift duties cause time-based conflicts, which create significant challenges for parents and children to spend time together, possibly resulting in problems among youths [6]. Likewise, husbands and wives see each other infrequently. However, regular family activities with the participation of every family member are crucial for family harmony [2], and therefore insufficient participation because of work may cause relationship crises. Besides, a reverse life schedule may lead to another type of work-family conflict, that is, strain-based conflict, which occurs when stress that arises from one role is carried or transferred to another role, which consequently results in symptoms of strain (such as anxiety and irritability), thus reducing effectiveness in the second role [2]. Sleeping is cited as most frequent activity for casino workers after work itself [12]; time spent with family is therefore sacrificed. Of particular significance, however, is that most casino employees reported both physical and psychological strains resultant of working in casinos, as revealed in previous interview studies [13]. The aforementioned research has also indicated that the need to display a welcoming attitude toward customers even if the customers are physically or verbally aggressive, has constituted into another considerable source of stress for casino dealers [7]. Dealers may display similar aggressive behaviors toward their family members and such a phenomenon has been well documented in the literature on emotion work [14]. This paper will therefore investigate whether the means that these dealers are using to handle their stress have an effect on their relationships with their spouse, children or other family members.

\section{THEORETICAL FRAMEWORK: MODEL OF WORK- FAMILY CONFLICT}

After a review of empirical research studies on workfamily conflict, Greenhaus and Beutell [2] proposed a related model. According to their model, there are three forms of work-family conflicts, namely, time-based, strain-based, and behavior-based conflicts. Time-based conflict occurs when time spent on one role makes it difficult to fulfill the requirements of another role. This conflict can take two forms; it can be either physically or mentally impossible to meet the expectations or demands of another role [2]. Timebased conflicts are directly associated with the amount of time spent on work and work schedule patterns, such as those that are regular or irregular, and flexible or inflexible. A parent who works long hours or night shifts and cannot care for his or her children is one such example. Strain-based conflict occurs when stress from one role affects the performance in another role. Role-produced strain, as 


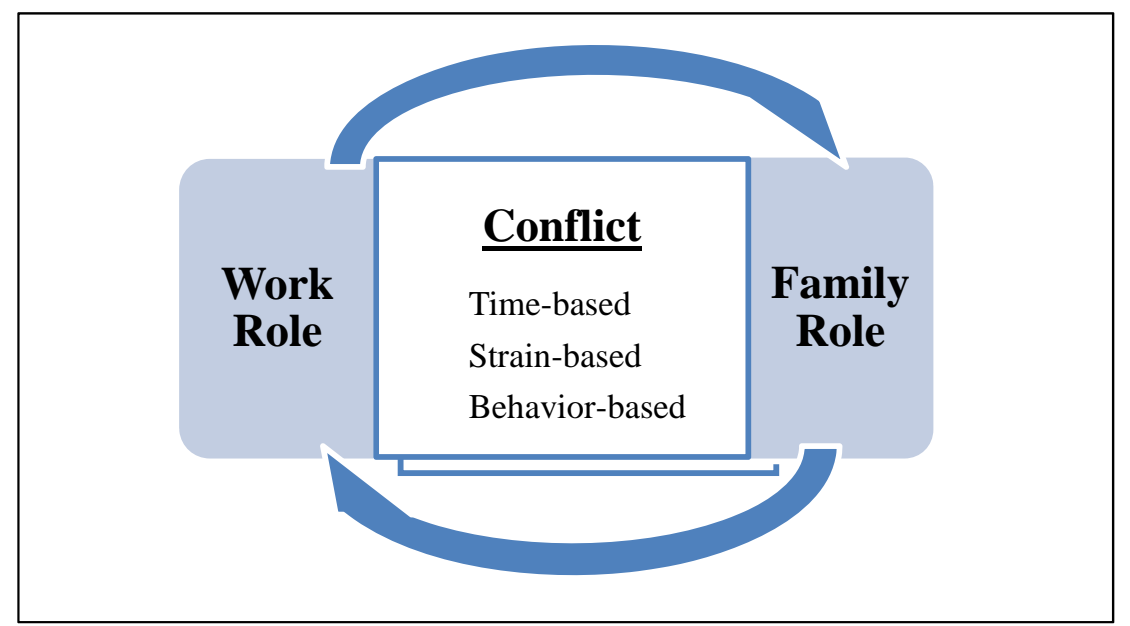

Fig. (1). Work-family conflict model.

generated from a variety of work stressors, may include symptoms such as fatigue, anxiety, and irritability. A typical example of strain-based conflict would be a stressful and exhausted individual who cannot perform the role and responsibility of a child caretaker at home. Finally, behaviorbased conflict occurs when an individual is unable to adjust his or her behavior to comply with the expectations of another role. For instance, a lawyer can be logical, objective, powerful and authoritative at work. However, s/he has to adjust his or her behaviors at home; otherwise, these may be incompatible with the behaviors desired by their spouse or children. Other factors, such as marital status, family structure, and couple employment pattern also play important roles in determining the level of work-family conflict. Although work can conflict with one's family life, it is worth pointing out that first, the relationship between work and family is bi-directional, so family life can also impose challenges to the work role. Second, work and family can be mutually supportive as long as there are no interferers between the work and family roles of an individual. Fig. (1) illustrates the conceptual structure of the work-family conflict model.

\section{METHODOLOGY}

\section{Design and Sampling}

A qualitative research method was employed to explore the impact of casino employment on the family relationships of dealers. The participants were recruited through the Gaming Teaching and Research Centre (GTRC) of the Macau Polytechnic Institute on a voluntary basis. The GTRC is a training centre for those who want to work in the tourism and gaming industries. All of the participants met the following criteria: (1) working as full-time dealers in a casino in Macau at the time of the interview, and (2) receiving or had undergone training at the GTRC or another such institute in Macau.

Ten focus group (Focus Groups A to J) interviews were conducted in Macau and each focus group involved 9 to 15 participants. There were a total of 113 participants who took part in the study. Each focus group interview was led by one of the three researchers who are involved with this study. The focus group interviews were conducted in a semistructured manner with guided questions (see Appendix A). This is to ensure the consistency of the information collected among the researchers. Written informed consent was obtained from each participant prior to the interview. The participants were informed of their anonymity, confidentiality of the responses, the voluntary nature of their participation and the right to withdraw at any point in time. Before the start of the interviews, the participants were asked to complete a demographic questionnaire (see Appendix B). The response rates were considered to be acceptable (see Table 1). The mean age of the participating dealers (101 of the 113 questionnaire respondents) was 32.5, which ranged from 19 to 55. The mean number of years in a dealer position was 4.2 years. The majority of the participants had completed secondary education, and had no children at the time of the completion of the questionnaire. About half of them were single.

\section{Data Collection and Analytic Approach}

All of the focus group interviews, which lasted from 60 to 90 minutes, were conducted in a meeting room at the GTRC, and audio-taped with consent from the participants. The tapes were then transcribed verbatim for coding analysis by using Nvivo-9 for the core themes. The codes were developed through preset and emergent categories [15]. Theme coding was undertaken by the researchers as well as an independent researcher who did not attend the focus group interviews. All of the transcripts were separately analyzed by following the six phases of thematic analysis as summarized by Braun and Clarke [16], including familiarization with the data, generating of the initial codes, searching for themes, reviewing the themes, defining and naming the themes, and producing the report. Core themes were then developed according to the types of relationships or their related conceptual ideas. Central themes were then synthesized after a joint discussion was held on the common themes. During the transcription and coding process, apart from the interviewers, all of the respondents were made anonymous by using pseudonyms. 
Table 1. Respondent profiles.

\begin{tabular}{|c|c|c|c|c|c|}
\hline Characteristic & Missing Data & Mean & SD & $n$ & $\%$ \\
\hline Age (Years) & 12 & 32.5 & 25.6 & 101 & \\
\hline Years of working as dealer & 6 & 4.2 & 4.8 & 107 & \\
\hline Gender & 0 & & & 113 & \\
\hline Male & & & & 55 & 48.7 \\
\hline Female & & & & 58 & 51.3 \\
\hline Education Level & 0 & & & 113 & \\
\hline Below Secondary & & & & 39 & 34.5 \\
\hline Secondary Graduate & & & & 47 & 41.6 \\
\hline Post secondary or higher & & & & 27 & 23.9 \\
\hline Marital Status & 3 & & & 110 & \\
\hline Single & & & & 59 & 52.2 \\
\hline Married & & & & 49 & 43.4 \\
\hline Divorced & & & & 2 & 1.8 \\
\hline Number of Children & 9 & & & 104 & \\
\hline 0 & & & & 64 & 61.5 \\
\hline 1 & & & & 21 & 20.2 \\
\hline 2 & & & & 17 & 16.3 \\
\hline 3 & & & & 2 & 1.9 \\
\hline
\end{tabular}

\section{FINDINGS}

The core themes of the effects of casino employment on overall family life were as follows: the financial situation of the family, conflicting schedules which influence family life, stress in the workplace, negative impacts on family relationships (spousal and parental), no impact on family relationships, and impacts on the younger generation from the perspective of the parents. These core themes are discussed in the following section, which will provide a significant understanding of the perspectives of these dealers in how their work has affected their family relationships and functions.

\section{Financial Situation of Family}

The perception of the respondents of the impact of casino employment on the financial situation of their family was positive. They all agreed that they are able to earn a higher income. The higher salary enables them to support their family needs, while at the same time, allows them to enjoy a more lavish lifestyle. Several participants expressed that the increased earnings have given them economic freedom, which in turn, have placed their family members more at ease. As depicted by John, one of the male respondents: "I am able to make a relatively steady and higher income from working in this industry, and because I have earned more, [my] living conditions have improved, [and] there is more enjoyment".

\section{Effect of Schedule Conflicts on Family Life}

Seventy-three of the respondents indicated that their shift work schedules have led to an imbalance between work and family life, which means that they have less time to see their family members and spend time with them. Aiden (a male participant in Focus Group J) pointed out that the conflict of his work time with that of his spouse has affected their relationship: "[We] communicate less as well. Sometimes when I go home after my shift, she is already sleeping. So I don't want to wake her up".

This conflict in schedule is found in couples who are both working as dealers as well as those with one spouse who is working in another industry. Brayden (a male participant in Focus Group C) said: "She [his wife] works from 9 a.m. to 5 p.m. as a clerk. It's like we are living in two completely different worlds.... I only have a limited amount of time to be with her ... and we have become more distant and less close to each other."

The conflict in schedules also affects parents and children. While the casino dealers are still sleeping, their children go off to school, and when the dealers return home after work, their children are already sleeping. Furthermore, children do not attend school on the weekends or Sundays, or during holidays and festivals, but casino dealers usually have to work regardless. Carey (a female participant in Focus Group I) stated: "It's very hard to get time off on a Sunday. We might be able to get a day off only after 
working for seven days.... In other words, we are only able to get a day off to spend time with our children on the weekend or a Sunday, if we have worked for forty-nine hours." Donna (a female participant in Focus Group E) concurred that: "... time spent with (my) children is reduced. When we have our holidays, our children need to go to school. [We] don't get to spend much time with them".

In summary, it is difficult for these dealers to see their family members. Erik (a male participant in Focus Group G) stated: "I can provide for my family, but I have lost time with them." In discussing the difficulties of finding the opportunity to see family members, Frazer (a male participant in Focus Group D) said: "I think it [the last meal with my family] was about 10 to 20 days ago.... [I] need to ask in advance to take time off as part of my annual leave to have dinner with my family." Several of the dealers interviewed for this study indicated that it was rare to be able to have meals with their families, particularly on special occasions.

\section{Stress at Work}

Fatigue is a common problem for dealers. Many respondents noted that their work requires a high level of concentration, and they are intensely afraid of making mistakes and losing their job. Gianna (a female participant in Focus Group B) said: "You have to be on the alert all of the time.... If you lose focus for just a while, then it's your fault!" Hannah (a female participant in Focus Group B) agreed: "Working as a dealer is already stressful ... it is even more stressful if you get a warning."

The stress and fatigue generated by work may affect the quality of sleep. Seventy-eight of the respondents reported sleeping problems, such as insomnia, and said that they feel exhausted all the time. Thus, they tend to spend their spare time on rest rather than an activity with their spouse, children or relatives. Iby (a female participant in Focus Group A) said: "I really do want to. I really want to [spend more time with her husband], but [I] really have no time and feel so tired..." Several of the respondents reported occasions when they had finished work and returned home during the day but felt too tired to supervise their children's schoolwork or respond to their children's request for their company. They only wanted to sleep. Jasmine (a female participant in Focus Group F) commented: "You don't get enough sleep, and then your kids ask if you can hang out with them. Of course not, [I'm] so tired that I don't think I can even walk." The importance of sleep and rest to the dealers means that they are unable to participate in family gatherings very often and have even less time to spend with family members: "There is less communication. My life is turned upside down. I spend more time sleeping ... see colleagues more often than family members" (Kyle, female participant in Focus Group G).

Stress at work also causes conflicts between family members. Fifty-nine of the respondents revealed that quarrels and other conflicts are often caused by the noise made by someone while the other person is sleeping. Laura (a female participant in Focus Group B) reported: "Makes noises when he [her husband] comes home ... wakes me up when I hear the noise. [I] don't get enough sleep and become irritated. [I] lose my temper and end up having an argument with him." Maya (a female participant in Focus Group H) reported the following related incident.

\begin{abstract}
Parents do indeed care about you, and I know this too, but sometimes you just can't tolerate them, like when they constantly make noises when you're sleeping. Then they want you to do this or that; actually, they just want to see you or talk to you. They will even wake me up for dinner when I don't want to get up, and so, that's how the conflicts come about.
\end{abstract}

\section{Negative Impacts on Family Relationships}

Less communication with the spouse, children and other family members is due, in part, to the aforementioned conflicting schedules and fatigue. Reduced communication and less quality time spent together are commonly seen by the respondents as destructive to their relationships. Nathan (a male participant in Focus Group G) commented on the interrelation between relationships and communication: "Less communication damages the bonds between spouses, and poor bonding causes them to spend less time with each other".

Some of the dealers may have more time together with a spouse who works in another industry, but even this may not necessarily improve their relationship. The two may find that they lack common ground because of their work in different industries and the lack of regular daily interaction. Carey said, "My husband and I are not in the same industry. Sometimes, I tell him about what I saw at work, but he doesn't understand what I am talking about."

Some of the respondents said they feel that their relationship with their children is unsatisfying or distant because they are not able to spend much time with them. Even when they have time off on a non-school day, their children do not have much to say to them. In addition, some of the respondents reported that they rely on grandparents or domestic helpers to help them raise their children, and consequently, their children have a closer relationship with their care givers rather than with them. Owen (a male participant in Focus Group A) recalled an incident that occurred when his son, whose caretaker is his grandmother, was 2 or 3 years old: "he didn't want to go home with us. Once he woke up during the night and was crying to go back to grandma's place. He couldn't sleep, and we had to take him back to grandma". Less contact means fewer opportunities for these dealers to show their care and concern toward their children. When parent-child communication deteriorates, the parent-child relationship subsequently suffers.

Many of the respondents indicated that their relationship with their own parents has also deteriorated. Preston (a male participant in Focus Group E) said, "My relationships with other family members have worsened. I argue with my dad. I see my family members less than twice a month." Similarly, Quinta (a female participant in Focus Group C) said, "My relationships have become so diluted that I don't know what is happening among my family members." Another said that his topics of interest were very different from those of his family members. It seems as though they are no longer able 
to connect with one another, which leads to even less communication.

In short, the interviews suggest that the conflicts in schedules and fatigue have far-reaching consequences for the relationships of these dealers with their spouse, children and other family members.

\section{Absence of Impacts on Family Relationships}

However, not all of the respondents felt that their work as dealers has a negative influence on their marital relationship or relationships with other family members. Reece (a male participant in Focus Group B), for example, reported that his wife also works shifts at a 24-hour convenience store, and thus the aforementioned schedule conflicts - and resulting discord - are not found in their relationship. Most of those who indicated that there is no detected negative impacts on their marital relationships reported that their partner also works in shifts. One of the female dealers said that her husband works the same time shift, and so their work patterns coincide. Sean (a male participant in Focus Group E) actually saw a positive side of spending less time together, and noted that there are fewer arguments: "Less time spent together, indeed, improves family harmony with one's spouse and parents".

Eighteen of the respondents also reported that they are unaware of any negative influences on family relationships because all of their family members work in the gaming industry and have thus grown accustomed to seeing one another less often. Eight of them even expressed the belief that they are spending more time with their family since working in the gaming industry because of factors such as shorter work hours as opposed to the work hours of previous jobs. Tyler (a male participant in Focus Group C) echoed this sentiment: "I used to work long hours. I started work at 12 p.m. and got off at 1 a.m.... Now, I work eight hours, so I have more time." However, it should be noted that all eighteen respondents who do not see negative influences on family relationships are single, while forty-three out of the forty-nine married respondents, especially those with children, reported negative influences, especially on their relationships with family members.

Twenty-one of the respondents felt that the positive effect of earning a higher income outweighs the negative effect of limited time with family. Ulrich (a male participant in Focus Group G) said, "[Life] is affected if there is no money; it is not affected if there is money.... I have to be realistic ... I need to make a living for my family." For ten of the respondents, family relationships are secondary to sufficient and higher income, although most still regarded effective communication with their spouse and family members as very important. All of the respondents agreed that working as dealers definitely reduces the time available for interaction and communication with their spouse, but that the effects on marital and other family relationships are not inevitably negative. Different dealers have different experiences, and utilize different coping methods. Some said that they try to interact with their spouse and other family members in their spare time, whereas others try to show understanding and make allowances for one another. No explicit negative effects were evident among those who adopted such relatively proactive approaches. In fact, their coping mechanisms on how casino employment impacts employees and, in turn, their relationships, are interesting, and warrants further research in future studies.

\section{Impacts on the Younger Generation from Perspective of Parents}

The respondents reported many parenting difficulties and, directly or indirectly, revealed their concern about the possible effects of their work patterns on their children. They have employed various strategies to reduce the negative effects. For instance, some of the couples who both work in the gaming industry choose to work different shifts to take turns as caretaker of their children, which, in turn, reduces the amount of interaction and communication that they carry out with each other. Moreover, even after making such adjustments, stress at work and schedule conflicts mean that these couples still have little time to spend with their children, thus making it very difficult to supervise their schoolwork and behaviors. Some of the respondents said that they find themselves becoming less tolerant of their children. Venea, a female dealer from Focus Group C, reported, "I'm not as tolerant as I was before. For example, if my son made a small mistake, I would try to be tolerant at first ... [sigh]... but, in the end, I [find that I] need to wake up and scold him."

Many of the respondents revealed that they find it difficult to follow the progress of their children at school, and some choose to send them to tutorial schools. Some of them indicated that their children even prefer that they are not at home to supervise their homework, as they can consequently avoid any parental discipline.

To compensate for the lack of time spent with their children, some of the respondents will buy toys for them and strive to satisfy their material wants in an effort to please the children. Erik said, "I [don't] give him [his son] lots of money, but I will buy what he asks for. He has lost his mobile three times, and I bought him three in one month." Although such material compensation appears to be common among the respondents with children, some said that they do not believe that it is beneficial, and noted that it might even develop undesirable levels of materialism in their children.

Finally, the respondents generally agreed that their children often display negative emotions. Whitley (a female participant in Focus Group H) said that her child is less happy than before: "S/he says "Mom always needs to work. No work. No work"'. Similarly, Xenia (a female participant in Focus Group C) reported: “... there are school functions that require the participation of the parents... but it's hard for us to get time off. [My child] isn't happy if [I] can't make it." Zachary (a male participant in Focus Group E) said that his son's grades are not very good, which prompts him to frequently ask his child if anything is wrong and the reason for his lack of effort in his studies. As a consequence, his son complains that his father is critical of him all the time.

It is evident that there is a direct relationship between the negative impacts of work as a dealer and the parent-child dyad. 


\section{DISCUSSION}

In this qualitative study, the aim is to explore the impact of casino employment on the family life of the dealers; in particular, their spousal and parental relationships. The findings obtained from the focus group interviews indicated that casino employment in general has positive economic impacts on the families of the participants. However, the participants also reported time-based and strain-based conflicts between their work and family roles. The most frequently mentioned problems are the conflicts between work and family life schedules, and the physical and psychological stress and strain induced by casino work. These findings echo those of previous studies that casino employees have relatively high stress levels [7], low degrees of job satisfaction [8] and experience fatigue [12]. Their mental health, which may be negatively affected by the stress that arises from work and deteriorating family relationships, and the resulting sleep and fatigue problems, is an issue of immediate concern. The mental health of these dealers and their family relationships are clearly interrelated, with one inevitably affecting the other. Further investigation is needed to arrive at a more thorough understanding and provide suitable preventive measures and interventions for these dealers and their families.

Besides, although some have reported that there is no impact on their family relationships, many find it challenging to reconcile the demands of work and child rearing. Shiftwork and stress at work seem to impose significant challenges to family functions and in many cases, even disrupt the performing of their family role. These conflicts can negatively affect the functioning and harmony of family life in Macau society in the long run. Dealers who are parents with the tendency to compensate their children with material goods, might unintentionally spoil their children and even instill materialistic values into them. In some cases, ineffective communication, and reduced family time between family members might cause further conflict between them.

Can the financial benefits of the gaming industry offset such harm to dealer families? If not, then what actions can the MSAR government, casino enterprises and the social system undertake to address these issues? Recommendations to address the three issues that most affect family relationships are discussed as follows.

\section{Addressing Schedule Conflicts}

The lack of communication and interaction with the spouse, children and other family members was considered as a consequence of the shift duties undertaken by the casino dealers. If shift duties are allowed as an option rather than made a compulsory requirement, this could ease time-based conflicts, according to Greenhaus and Beutell [2]), and such a policy has been already implemented in the casino enterprises of Las Vegas and Monte Carlo. Hing and Breen [17] similarly proposed that casino enterprises should allow for more regular shifts, in that dealers can have the flexibility to choose their preferred schedules, in accordance with those of their family, so that they can spend more time with them and thus enjoy a well-functioning family life. In accordance with the work carried out by Lambert et al. [18], providing dealers with the flexibility to plan their schedules and accommodating their agendas for both family life and work are essential if the negative effects discussed in this paper are to be minimized.

\section{Addressing Family Harmony}

It is recommended that casinos could hold special events, such as carnivals and workshops, for the enjoyment of the families of the dealers so as to improve family harmony and restore a well-functioning family life. Such family events could provide the participants with the opportunity to spend time together and enhance mutual understanding. Hing and Breen [17] suggested that the promotion of these types of family and social activities can relieve gambling-related issues among casino employees, which has been fully implemented in Las Vegas and Monte Carol for a period of time. As well, family and social activities provide the opportunity for family members to see one another more often, and help to reduce time-based conflicts and build family resources which prevent casino employees from indulging in gambling themselves.

To ease strain-based conflict, it is suggested that casinos offer educational workshops to their employees. The focus of these workshops could be on differentiating between work and home identities, equipping employees with more effective ways of handling different family issues, and helping them to improve their communication and interactions with their spouse, children and other family members. Moreover, counseling services or support groups could provide casino employees with the psychological support that would help them to address work stress, thereby reducing the adverse effects of strain-based conflicts [18].

According to Leung [19], several organizations have approached casino enterprises in Macau to provide counseling services, educational programs or support groups for their casino employees, but there is, as yet, no clear outcome. Support from the government, casino enterprises and the social system is clearly needed to carry out such programs to achieve optimal outcomes for casino employees, their families and society as a whole.

\section{Consideration of Children and Teenagers}

It is vital that multi-disciplinary support be provided to the families of dealers. It is also necessary for schools and other sectors of society to assist children whose dealerparents may not have sufficient time to address their needs. Remedial classes to address academic issues, counseling sessions to address personal issues and programs aimed to enhance relationships with peers, family members and teachers are recommended. Day care centers could also be established near the casinos for the children of their employees, as is the case in other countries. Support and guidance from teachers [20] and school-based prevention programs [21] have also been shown to be effective in helping students stay in school and alleviating other problems, such as drug abuse and gambling. Such support is designed to deal with the aftermath of the malfunctioning family systems that result from time, strain and behavior based conflicts between work and family. 
The casino enterprises themselves also need to assume more responsibility [19]. All of the aforementioned types of support require close cooperation among the government, casino enterprises and the relevant social systems for the families of dealers, the younger generation and society as a whole.

The MSAR government must deal with the range of issues that have arisen resultant of the position of Macau as a gaming city from both macro- and micro-perspectives. Although the casino enterprises must undeniably bear the responsibility of providing support to their employees and their families to minimize the adverse influences on their well-being, the MSAR government is also responsible for ensuring that there is balance between the work offered by these enterprises and the personal life of their employees to achieve peaceful and harmonious conditions for the continual healthy development of Macau's economy and the livelihood of its people.

\section{LIMITATIONS}

This study has two major limitations. First, the sample size is relatively small. There are an estimated 48,600 dealers in Macau. Hence, the 113 dealers who participated in this study represent just $0.23 \%$ of the total dealer population. Second, in addition to dealers, other gaming industry employees, such as supervisors, cleaners, servers and so on, are also likely to be affected by the casino culture, although their voices are not represented in this study. Despite these significant limitations, this study constitutes the first qualitative research that focuses on dealers, who comprise a large sector of casino employees, and the ways in which their jobs affect their family relationships. The findings will certainly raise awareness among the general public and serve as a platform for other qualitative research that intend to focus on the influence of casino work on family relationships. In response to the diverse opinions with regard to spousal, parental and overall family relationships, future research could include specific inclusive criteria of the participants so as to collect more in-depth information on the effects of the mentioned relationships. For instance, participants have to be married for investigation on spousal relationships, or have children for investigation on parental relationships, etc.

\section{CONCLUSION}

To conclude, the rapid development of the gaming industry has had both positive and negative effects on Macau. As previously noted, the profit derived from this industry is clearly beneficial for Macau's economy, and the development of the gaming industry has undoubtedly helped to reduce unemployment by providing many job opportunities. However, the possibility of negative influences means that the MSAR government and the public need to address the related issues. The findings of this study illustrate that the work of dealers may create the work-family conflicts outlined by Greenhaus and Beutell [2]: time-based and strain-based conflicts. It is not only the 83,300 gaming industry-related employees who are influenced by the work culture of the gaming industry, but their family members as well. The long-term consequences are borne by the individual casino employees, their families and society. It is important to more frequently provide dealers with a voice and determine in more depth, the influences of their work on them and their fellow casino employees and families.

The ecological context of human living conditions plays an important part in shaping human behaviors and understanding them. Macau, with a distinct culture resultant of the fusion of the Portuguese and Chinese cultures, and the expansion of the gaming industry, has its unique contextual features that is worthy of our attention. This evidence-based, indigenous in content and culturally sensitive study explores the specific ways in which family relationships have changed among Macau families with casino dealers and how they have been affected by the socioeconomic changes brought about by casino employment. The perspectives drawn from the casino dealers in Macau can furnish insights into the hidden problems that are associated with the gaming industry, especially in parent-child relationships.

\section{CONFLICT OF INTEREST}

The authors confirm that this article content has no conflict of interest.

\section{ACKNOWLEDGEMENTS}

The project was funded by the Faculty Research Grant of Hong Kong Baptist University.

\section{APPENDICES}

\section{Appendix A: Focus Group Interview Guidelines}

1. Would you please describe your parental relationship?

2. How often do you spend time with your child/children?

3. Do you think that you know your child/children well?

4. How often do you give your child/children an allowance and how much do you give?

5. Do you think that the amount is adequate?

6. What do you do in your spare time?

7. What does your child/children do in his/her/their spare time?

8. Do you think that your job has had an impact on your family life? If so, what are the impacts?

9. Why have you chosen this career?

10. Is there anything lacking in your relationship with your family members?

11. Does your career have any positive influences on your family?

12. How much leisure time do you have each week? Do you think that it is sufficient?

13. What do your various family members think about gambling? Do they think the same way that you do? 


\section{Appendix B: Profile of Macau Casino Employees}

Name: Age:

Education Level: Job (Nature):

Years Working in Gaming Industry:

Marital Status: Single/Married/Divorced

Number and Age(s) of Children:

1) What were your reasons for joining the gaming industry?

2) How many hours do you usually work each week?

3) Are you responsible for any of the following household duties?

$\square$ Cooking $\square$ Child rearing $\square$ Domestic chores

$\square$ Emotional support of your family members $\square$ Other:

4) How many hours (per week) do you spend/spend on communication with your family (children, parents or spouse)?:

5) Please briefly describe your thoughts/feelings toward your job:

6) Please briefly describe the thoughts/feelings of your family members toward your job:

7) Would you choose to continue your job if you had a choice?

Yes:

$\square$ No:

We sincerely invite you to take part in an individual interview after the focus group discussion. Would you be willing to attend an individual interview? Yes/No

If yes, then please provide us with the following information:

Contact number: E-mail:

Thank you very much for the information provided, which will be kept confidential and strictly used for academic research purposes. Should you have any inquiries, please feel free to contact XXX at XXXX XXXX (telephone number).

\section{REFERENCES}

[1] Korn DA, Gibbins R, Azmier J. Framing public policy towards a public health paradigm for gambling. J Gambl Stud 2003; 19(2): 235-56.

[2] Greenhaus JH, Beutell NJ. Sources of conflict between work and family roles. Acad Manage Rev 1985; 10(1): 76-88.

[3] Statistics and Census Service of Macau Special Administrative Region Government. Yearbook of statistics [Internet]. Macau SAR: Statistics and Census Service 2003 [cited 2014 Feb 9]. Available from: http://www.dsec.gov.mo/Statistic.aspx?NodeGuid=d45bf8ce2b35-45d9-ab3a-ed645e8af4bb\#P9205

[4] Statistics and Census Service of Macau Special Administrative Region Government. Yearbook of statistics [Internet]. Macau SAR: Statistics and Census Service 2013 [cited 2014 Nov 26]. Available from: http://www.dsec.gov.mo/Statistic.aspx?NodeGuid=d45bf8ce2b35-45d9-ab3a-ed645e8af4bb\#P19898

[5] Gaming Inspection and Coordination Bureaus of Macau SAR (n.d) [Interent]. Macau SAR: Gaming Inspection and Coordination Bureau 2013 [cited 2013 Nov 26]. Available from: http://www.dicj. gov.mo/web/en/history/index.html
[6] Macau SAR. Report on the youth's problems and the development of social service in Macau (in Chinese). Macau SAR: Social Welfare Bureau of Macau Special Administrative Region Government 2005.

[7] Zheng X, Luo M, Tao W. On the job stresses of employees in Macau's gaming industry (in Chinese). J Macau Poly Ins 2009; 1 : 15-26.

[8] Dangerfield L. Job satisfaction, substance abuse, and gambling behavior of northern Albertan casino employees (Unpublished Master thesis) [Internet]. Alberta: University of Lethbridge 2002 [cited 2013 Nov 26]. Available from: http://www.uleth.ca/dspace/ bitstream/10133/553/1/dangerfield\%2c\%20lyndsey.pdf

[9] Hing N, Breen H. Workplace factors that encourage and discourage gambling amongst gaming venue employees: A managers' perspective. Int J Ment Health Addict 2007; 5(4): 346-66.

[10] Lee T K, LaBrie R A, Rhee HS, Shaffer HJ. A study of South Korean casino employees and gambling problems. Occup Med-c 2008; 58(3): 191-7.

[11] Trout D, Decker J, Mueller C, Bernert JT, Pirkle J. Exposure of casino employees to environmental tobacco smoke. J Occup Environ Med 1998; 40(3): 270-76. 
[12] Frey JH, Carns DE. Job satisfaction of casino card dealers. Sociol Soc Res 1988; 72(3): 159-64.

[13] Wong LK, Lam PS. Work stress and problem gambling among Chinese casino employees in Macau. Asian J Gambl Iss Pub Health [Internet]. 2013 (cited 2015 Feb 10); 3:7. Available from Springer Link: http://www.ajgiph.com/content/3/1/7

[14] Hochschild AR. The managed heart: commercialization of human feeling. Berkeley and Los Angeles, California: University of California Press 1983

[15] Hathaway RS. Assumptions underlying quantitative and qualitative research: Implications for institutional research. Res High Educ 1995; 36(5): 535-62.

[16] Braun V, Clarke V. Using thematic analysis in psychology. Qual Res Psy 2006; 3: 77-101.
[17] Hing N, Breen H. Working in Australian gaming venues, and shiftwork. J Hosp Manag 2008; 15(1): 8-14.

[18] Lambert EC, Hogan NL, Camp SD, Ventura L A. The impact of work-family conflict on correctional staff: A preliminary study. Crim Crim Justice Int J 2006; 6(4): 371-87.

[19] Leung KY. Macao family education: The challenge of balancing work and family. J Youth Stud 2010: 13(1): 156-64.

[20] Croninger RG, Lee VE. Social capital and dropping out of high school: Benefits to at-risk students of teachers' support and guidance. Teach Coll Rec 2001; 103(4): 548-81.

[21] Eggert L, Seyl C, Nicholas LJ. Effects of a school-based prevention program for potential high school dropouts and drug abusers. Int $J$ Addict 1990; 25(7): 773-801.

(C) Chan et al.; Licensee Bentham Open.

This is an open access article licensed under the terms of the Creative Commons Attribution Non-Commercial License (http://creativecommons.org/licenses/by-nc/3.0/) which permits unrestricted, non-commercial use, distribution and reproduction in any medium, provided the work is properly cited. 